\title{
Dynamics of Moving Electron Vortices and Magnetic Ring in Laser Plasma Interaction
}

\author{
D.N. Yue, ${ }^{1,2}$ M. Chen, ${ }^{1,2, a)}$ P.F. Geng, ${ }^{1,2}$ X.H. Yuan, ${ }^{1,2}$ S.M. Weng, ${ }^{1,2}$ S.S. Bulanov, ${ }^{3}$ S.V. Bulanov, ${ }^{4,5, b)}$ K. \\ Mima, ${ }^{6}$ Z.M. Sheng, ${ }^{1,2,7,8}$ and J. Zhang ${ }^{1,2,9}$ \\ 1) Key Laboratory for Laser Plasmas (Ministry of Education), School of Physics and Astronomy, \\ Shanghai Jiao Tong University, Shanghai 200240, China \\ ${ }^{2)}$ Collaborative Innovation Center of IFSA, Shanghai Jiao Tong University, Shanghai 200240, \\ China \\ 3) Lawrence Berkeley National Laboratory, Berkeley, California 94720, USA \\ ${ }^{4)}$ Institute of Physics of the ASCR, ELI Beamlines Project, Na Slovance 2, 18221 Prague, \\ Czech Republic \\ ${ }^{5)}$ Kansai Photon Science Institute, National Institutes for Quantum and Radiological Science and Technology, \\ 8-1-7 Umemidai, Kizugawa, Kyoto 619-0215, Japan \\ 6) The Graduate School for the Creation of New Photonics Industries, Hamamatsu, \\ 431-1202 Japan \\ 7) SUPA, Department of Physics, University of Strathclyde, Glasgow G4 ONG, \\ United Kingdom \\ 8) Tsung-Dao Lee Institute, Shanghai 200240, China \\ 9) Institute of Physics, Chinese Academy of Sciences, Beijing 100080, China
}

(Dated: 16 March 2021)

\begin{abstract}
Moving electron vortices have been observed in laser interaction with non-uniform near-critical-density plasma by multi-dimensional Particle-in-Cell simulations. In two dimensional geometry there are two vortices with opposite magnetic polarity, moving perpendicularly to the plasma density gradient direction. The field distribution and particle motion composing such moving structure have been clearly observed in simulations, which explains the vortex motion. Two components of loop currents are formed around each electron vortex, which dominate the vortex motion. The moving velocity can be as large as $0.2 c$ level, forming relativistic vortices inside the plasma. Laser plasma conditions such as intensity, polarization, density profile and external magnetic field effects on the vortex motion and evolution are also studied. In three dimensions, the structure appears as an expanding magnetic ring with an internal magnetic field up to 1000 Tesla. Such vortex structures suggest an interesting way of energy (with more than $5 \%$ of the laser energy) transportation to ambient plasmas as far as $50 \mu \mathrm{m}$ away from the laser-plasma interaction region, which may have applications in laser plasma based inertial confinement fusion and laboratory astrophysics.
\end{abstract}

\section{INTRODUCTION}

Nonlinear structures such as solitons, vortices and collisionless shock waves are widely known in various areas. Studies on these structures are interesting and important both for fundamental researches and applications, such as understanding of high energy particle acceleration in universe or reducing the damage of tornadoes. Along with the recent rapid development of intense laser technology ${ }^{1,2}$, such kind of structures can be generated and studied in detail in intense laser plasma interactions. Among them electron vortices formed in relativistic laser plasmas have recently aroused broad interests ${ }^{3-8}$ due to their wide presence in nature and potential applications in the laser electron and ion acceleration ${ }^{9-21}$, as well as extremely strong magnetic field generation ${ }^{22,23}$. Compared with normal target sheath field in laser solid interaction, in addition to ion acceleration, the vortex field can also be used to collimate the accelerated ions ${ }^{11}$, and gen-

\footnotetext{
a) minchen@sjtu.edu.cn

b)Sergei.Bulanov@eli-beams.eu
}

erate quasi-monoenergetic protons with energy of hundreds of $\mathrm{MeV}^{21}$.

In some other studies, it has already been found that in a non-uniform plasma when the plasma density scale length $L_{n}$ is far smaller than the collisionless skin depth of ions (i.e. $L_{n}=\left|n_{e} / \nabla n_{e}\right| \ll c / \omega_{p i}$, where $n_{e}$ is the electron density, $c$ is the speed of light and $\omega_{p i}$ is the ion Langmuir frequency), electron vortex expands and its center also moves. This type of electron vortex has been discussed in details in the case of plasma-opening switches, Z-pinches ${ }^{24,25}$ and some laser plasma interaction studies ${ }^{10}$. The center speed that electron vortex moves along the direction perpendicular to the plasma density gradient direction is known as the Hall speed which can be derived from Electron Magnetohydrodynamics (EMHD) equations ${ }^{25,26}$. If the ion motion effects are negligible, the center speed fits well with the Hall speed, otherwise, the center speed becomes smaller ${ }^{25,27}$.

In this paper, by using multi dimensional Particle-inCell (PIC) simulations, we study the generation and dynamics of vortex structures during laser interaction with nonuniform plasmas with near critical density. We see that in two dimensional (2D) geometry there are bipolar magnetic field structures moving with electron vortices 
with center speed up to $0.23 c$, which is much larger than the normal Hall speed. And we also found that the electrons moving around the vortex are relativistic. It shows that a relativistic vortex structure exists in laser near critical density plasma interaction. In three dimensional (3D) geometry, we find that an expanding magnetic ring with an internal magnetic field up to 1000 Tesla can be generated. The magnetic field reduces as the ring expands, which is much faster than the normal ion motion induced magnetic field dissipation in the case of standard electron vortices ${ }^{25}$. These studies provide new findings and observed characters on the dynamics of magnetic vortex structure and are helpful to understand the energy transportation during laser near critical density plasma interaction.

\section{STRUCTURE OF RELATIVISTIC ELECTRON VORTEX}

The relativistic PIC code OSIRIS ${ }^{28}$ has been used to perform multi dimensional simulations. A laser pulse with polarization along $y$ direction and wavelength of $\lambda_{0}=0.8 \mu \mathrm{m}$, peak intensity of $I_{0}=3.43 \times 10^{19} \mathrm{~W} / \mathrm{cm}^{2}$ propagates along $x$ direction, corresponding to a normalized laser electric field $a_{0}=4.0$. The pulse takes Gaussian profiles both in longitudinal and transverse directions with FWHM pulse duration of $10 \mathrm{fs}$ and FWHM focus spot size of $6 \mu \mathrm{m}$. The simulation boxes are $34 \mu m \times 100 \mu m$ for $2 \mathrm{D}$ and $34 \mu m \times 34 \mu m \times 34 \mu m$ for $3 \mathrm{D}$ simulations. The boxes are divided into $680 \times 2000$ and $544 \times 544 \times 544$ cells for $2 \mathrm{D}$ and $3 \mathrm{D}$ simulations, respectively. Such simulation boxes are large enough to ignore nonphysical boundary effects during the whole simulation window, which is confirmed by another simulation where the box size has been extended to $100 \mu m \times 100 \mu m$. A plasma with plateau-downramp-plateau density profile is placed at the region of $10 \mu m<x<34 \mu m$. The plasma density is uniformly distributed both along $y$ and $z$ directions. Along the $x$ axis, the density of the first plateau equals $0.24 n_{c}$ within the regions of $10 \mu m<x<$ $18 \mu \mathrm{m}$. The length is chosen to be smaller that the depletion length of the laser pulse in near critical density plasma ${ }^{13,15}$, which is about $9 \mu m$ for the given parameters. Then the plasma linearly decreases to $0.032 n_{c}$ within $3 \mu \mathrm{m}$. In the second plateau region it is equal to $0.032 n_{c}$ as shown in the inset of Fig. 1(a). Here $n_{c}=1.74 \times 10^{21} \mathrm{~cm}^{-3}$ represents the critical density for the drive laser.

In the simulation, we find when the ultrashort laser pulse propagates from the high density region to the low density region, around the density downramp part, there are two electron vortices formed on both sides of the laser propagation axis. In 2D geometry, each of the vortices can carry about $5 \%$ of the drive laser energy and move transversely. The vortex dissipates its magnetic and electric energies to ambient plasmas along the path. In this simulation, the energies have been transported to
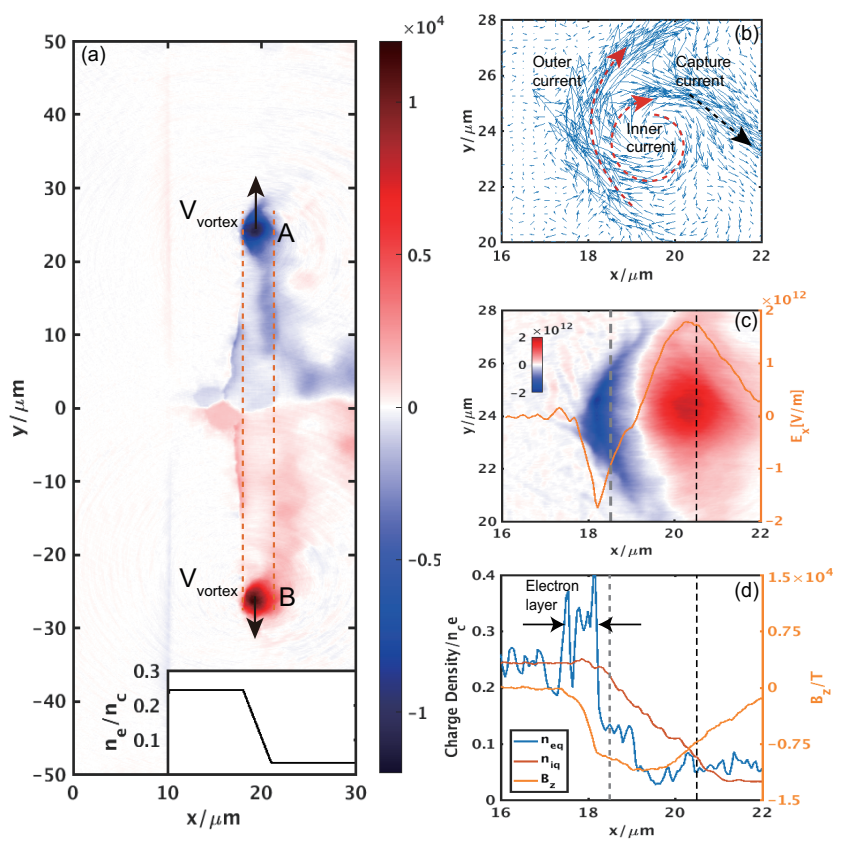

FIG. 1. (a) Distribution of magnetic dipole structure (magnetic field $B_{z}$ component in the unit of Tesla) and initial plasma density (shown in the inset figure). (b) Distribution of the electric current density vector around the vortex A at $t=150 T_{0}$. (c) Distribution of the asymmetric longitudinal electric field $E_{x}$. (d) Distribution of electron and ion charge density $\left(n_{e q}\right.$ and $\left.n_{i q}\right)$, magnetic field $\left(B_{z}\right)$ associated with the vortex A at $y=24 \mu \mathrm{m}$ cutting through the center of the vortex at $t=150 T_{0}$.

a region as far as $50 \mu m$ away from laser axis where the laser pulse cannot reach. Typical electric and magnetic fields, electric current, and plasma density distributions after the laser passing through the downramp plasma are shown in Fig. 1. Two magnetic field structures with opposite polarity moving with two electron vortices are observed as shown in Fig. 1(a). The magnetic fields are along $z$ direction and have similar maximum intensities $\left|B_{z}\right| \sim 10^{3} T$. These fields are induced by loop currents inside the vortex as shown in Fig. 1(b). One can see that the currents are composed of three different parts. The outer one $\vec{j}_{\text {out }}$ shows a half ring structure and the inner one $\vec{j}_{\text {in }}$ has a full ring structure. The last one is the capture current which is composed by electrons from the low density region. These electrons are trapped by the electromagnetic fields of the vortex and some of them finally compose the inner current.

Within each vortex, besides the magnetic fields there are also radially directed electric fields. The $E_{x}$ component of the electric fields is shown in Fig. 1(c). The orange line shows the $E_{x}$ distribution along the line of $y=24 \mu \mathrm{m}$. As one can see that the $E_{x}$ distribution is asymmetric around the zero point which is more close to the left side. The charge density distribution shown in Fig. 1(d) gives hints of such asymmetry. The ion distri- 
bution does not vary so much during the vortex transversely passing by. It keeps the initial density downramp profile. However, electrons at the high density region (around $x=18 \mu \mathrm{m}$ ) have been compressed, forming a higher density layer. This asymmetric charge separation leads to the electric field distribution showing an eccentric circle shape. The center velocities of the two vortices are measured to be $0.23 c$ which is close to the relativistic level. Such high speed vortex has also been reported recently by Yi et. al. when they studied the ion acceleration driven by a moving electron vortex ${ }^{21}$ with much higher laser intensity and different laser incidence direction. In our case the laser intensity is orders of magnitude smaller and there is no efficient ion acceleration observed in front of the vortex. We focus our studies on the vortex motion itself. To figure out the dynamics of the vortex, we firstly investigate the single electron motion inside and around the vortex.

\section{DYNAMICS OF ELECTRON VORTEX IN TWO-DIMENSIONAL GEOMETRY}

\section{A. Microdynamics of the moving electron vortex}

Two different sets of electron motion, forming the outer and inner currents, are observed as shown in Fig. 1(b). They appear in the density downramp region. Electrons labelled with green, yellow and blue colors shown in Fig. 2(a) make the first kind of motion and do not move with the vortex as their start and end points indicate. As a whole they make downward motion and induce the local outer current which induces the vortex magnetic fields. These motions are due to the transverse charge separation force in the high density region. The second kind of electrons have a much larger transverse drift and make an upward motion as shown by the orange curve in Fig. 2(a). Besides the whole drift motion, all the electrons undergo local clockwise rotation. To show the difference of these two kinds of motion clearly, we transform the electrons trajectories to the frame moving with the vortex drift velocity. We line out the transformed trajectory $\left[x(t), y(t)-V_{\text {vortex }} t\right]$ on a vortex background fields as shown in Fig. 2(b). Obviously one can see that the first kind of motion is just once pass by the vortex as shown by the downward trajectory $L_{2}$ with start points $i_{2}$ and end point $f_{2}$. The second kind of electron motion has multi periodic motions around the vortex boundary as shown by the circular trajectory $L_{1}$ with the start point $i_{1}$ and the end point $f_{1}$. These electrons mainly come from the central downramp region where the laser propagates from the high to low density region. These electrons are initially pushed forward by the laser ponderomotive force and then are pulled back by the local charge separation field. The process looks like an electron fountain. In principle, the behavior of the laser pulse in high density region can be used to estimate the initial magnetic field of the vortex. As the laser pulse propagates through that region, it creates a channel in plasma density and generates a strong electron current along the propagation axis ${ }^{13,15,19}$. This current eventually becomes pinched amplifying the magnetic field in the channel. The maximum strength of the magnetic field according to the results of Reference ${ }^{19}$ is proportional to the square root of laser power and is approximately equal to $2 \times 10^{3} \mathrm{~T}$ for the laser parameters used here. This is in good agreement with the results of PIC simulations. Along the route of vortex transverse motion some peripheral electrons are also involved in the vortex formation and contribute to the inner current which further enhances the magnetic field.
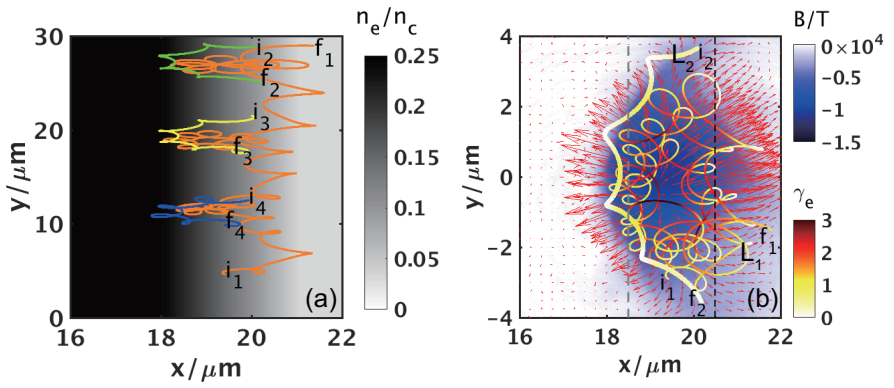

FIG. 2. Typical trajectories of electrons contributing to the electric currents around the vortex. Here $i_{1}, i_{2}, i_{3}$, and $i_{4}$ represent the initial positions of four selected electrons and $f_{1}, f_{2}, f_{3}$, and $f_{4}$ represent their final positions. (a) shows trajectories in the laboratory frame and (b) shows trajectories in the frame moving with the vortex. The background in (a) is the initial plasma density distribution. The red arrows in (b) represent the electric field vector and the background is the spatial distribution of magnetic field.

For $L_{1}$ trajectory, they are actually composed of two types of distinguishable sub-motions. The first one is small-scale clockwise Larmor motion due to the local magnetic field. The other one is an anticlockwise motion with larger radius. For the Larmor motion, due to the asymmetric distribution of magnetic fields, the Larmor radius of electrons on the right side of the vortex is larger than that on the left side. Besides the influence of magnetic fields, electrons are also subjected to the radial static electric fields as shown in Fig. 1(c). The combined effect will induce a Larmor motion center drift with velocity of $\vec{v}_{d r i f t}=\vec{E} \times \vec{B} / B^{2}$. This leads to the large scale anticlockwise motion. As one can see from the positions labeled by the vertical dashed lines in Figs. 1(c,d) and Fig. 2(b), the absolute value of magnetic field is larger and of the electric field is smaller at the gray dashed line labeled position compared with the fields at the black dashed line labeled position, so the speed of the downward drift at the left side is much smaller than the upward drift at the right side, which makes the vortex have a whole upward motion as shown in Fig. 2(a) by the orange curve. Obviously the $L_{1}$ motion also contributes to the inner electric current which further enhances the vortex magnetic field. Finally an upward moving vortex 
structure with magnetic field is generated.

\section{B. Energy dissipation of electron vortex}

Along with the transverse motion, the vortex interacts with ambient plasmas. Both the static electric and magnetic energies inside the vortex are dissipated during the interaction. In Fig. 3, the evolutions of such energies carried by vortex $\mathrm{A}$ in Fig. 1 are shown and they are fitted by linear lines. The energy dissipation rates can be obtained from these lines, i.e. $\epsilon_{b}=-1.6 \times 10^{-4} t+0.0473$ and $\epsilon_{e}=-4.9 \times 10^{-5} t+0.0143$ for electric and magnetic energies, respectively. As one can see, the magnetic field is more dominant than the electric field inside the vortex. These energies are dissipated away at $t \approx 300 T_{0}$ and the vortex disappears at $y \approx 50 \mu m$.
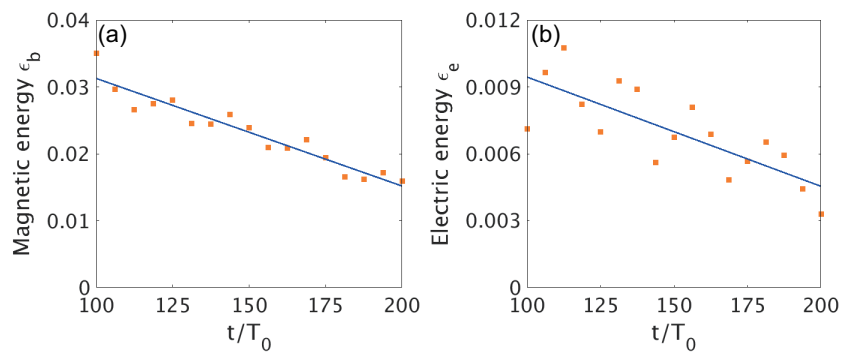

FIG. 3. Evolutions of the magnetic energy $\epsilon_{b}$ (a) and the electric energy $\epsilon_{e}$ (b) of vortex A in Fig. 1(a). The electric and magnetic energies are both normalized to the laser energy.

It is interesting to find that in $2 \mathrm{D}$ geometry, actually this kind of energy dissipation does not lead to the slower motion of the vortex. We traced the maximum magnetic field at the vortex center and found it varies quite slowly as shown in Fig. 4(a). It means that the magnetic energy dissipation is mainly by the vortex size reduction, not by weakening the magnetic field. For the vortex drift velocity, the energy dissipation has little effects. The other contribution term on the velocity, i.e. the inertial term, also varies slowly. As a result, the vortex velocity only slightly changes over the total simulation time and it has a uniform motion as shown Fig. 4(b).

\section{LASER-PLASMA EFFECTS ON VORTEX GENERATION AND DYNAMICS}

\section{A. Plasma density gradient and laser intensity effects}

In section III, we have analyzed the vortex motion through microdynamics. The vortex motion is also widely studied by using EMHD model ${ }^{24,25,27}$, where the displacement current effects are usually neglected. Such neglection is still valid in our case since the electromagnetic fields inside the vortex is at least one order of magnitude smaller than the static fields. Although in our sim-
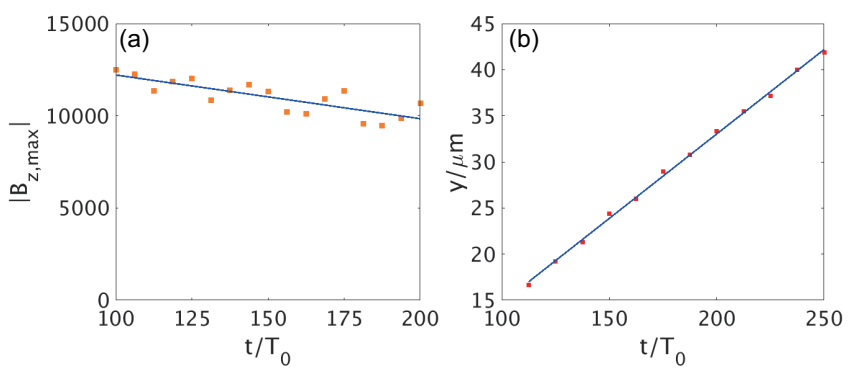

FIG. 4. (a) Evolution of the amplitude of the magnetic field at the vortex center of vortex A in Fig. 1(a). (b) The center positions of vortex $\mathrm{A}$ at different times.

ulation cases, the vortex moving velocity is larger than 0.1c, the following study shows the EMHD model still works well once the relativistic factor $(\gamma)$ is included in the inertia term. With these approximations, the evolution of the generalized vorticity of the electron vortex $\Omega_{z} \vec{z}=\nabla \times \vec{p}+e B_{z} \vec{z}$ satisfies the following equation ${ }^{27,29}$.

$$
\partial \Omega_{z} / \partial t+(\vec{v} \cdot \nabla) \Omega_{z}=-(\nabla \cdot \vec{v}) \Omega_{z}
$$

where $\vec{z}$ represents the unit vector and $\vec{v}$ is the velocity of electron fluid. From Eq. 1, one can deduce the vortex velocity as $\vec{V}_{\text {vortex }}=\Omega_{z} \vec{z} \times \nabla n_{e} / \mu_{0} n_{e}{ }^{2} e^{2}$. For normal cases the inertia term $\nabla \times \vec{p}$ can be negligible, the vortex velocity can be expressed approximately as the normal Hall speed, $\vec{V}_{H}=B_{z} \vec{z} \times \nabla n_{e} / \mu_{0} n_{e}{ }^{2} e^{26}$.

We have compared our simulated vortex velocities with these two estimated velocities for the cases of a serial laser plasma parameters with different plasma density gradient $\left(\nabla n_{e}\right)$ and laser intensity $\left(a_{0}\right)$. The vorticity $\left(\Omega_{z}\right)$ used in the theoretical calculation is directly obtained from the simulation results. We use such 'half-analysis half-simulation' calculation to see the effect of the inertial term. The results are shown in Fig. 5. As one can see that the Hall speeds underestimate the velocity for all the cases. The other estimated velocities with the inertial term contribution are close to the simulation results, which indicates that the vortex studied here is not a magnetic field dominated one. In further, as one can see from Fig. 5(b) that when the laser intensity $\left(a_{0}\right)$ increases, the usual Hall speed saturates but the simulated vortex speed continues to increase. The reason is that the electric current density tends to be saturated since it cannot be larger than $n_{e} e c$, which results in the saturation of the magnetic field $\left(B_{z}\right)$ and so on the Hall speed. However, in this case, the inertial term does not saturate because $\nabla \times \vec{p}$ is related to the relativistic factor $(\gamma)$ which increases with the laser intensity $\left(a_{0}\right)$. From the figure we can also infer that the vorticity is proportional to the drive laser intensity i.e. $\Omega_{z} \propto a_{0}$, suggesting that the carried energy including the magnetic field energy and electron kinetic energy are approximately proportional to the laser energy. These findings are quite different from the previous studies ${ }^{27,29}$. 

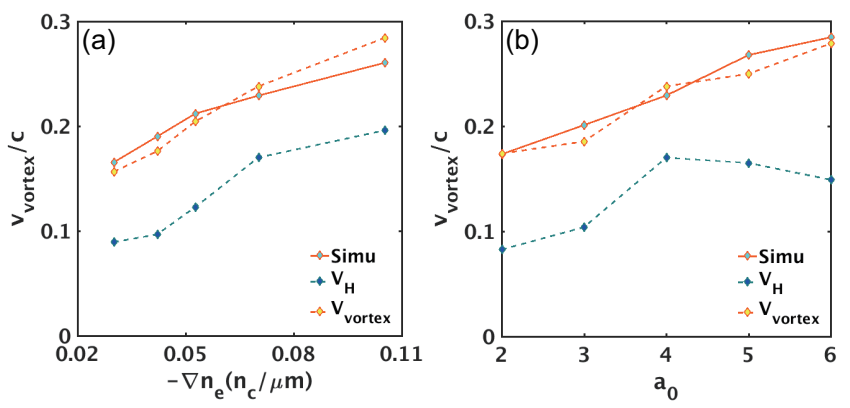

FIG. 5. Normalized Vortex velocity $V_{\text {vortex }} / c$ vs plasma density gradient $-\nabla n_{e}$ for $a_{0}=4.0$ (a) and laser amplitude $a_{0}$ for $-\nabla n_{e}=0.07 n_{c} / \mu m$ (b). The Hall speed, $V_{H}$, simulation results and the analytical result $V_{\text {vortex }}$ are all shown.

\section{B. Density profile effects}

In the above studies, a linear density downramp profile for the plasma has been used. We should point out that the vortex generation does not rely on such "plateaudownramp-plateau" profile. In realistic experiments, plasmas with exponential density profiles are usually generated by pre-pulse of high-contrast laser irradiating on a nanometer-thick foil target ${ }^{18,36,37}$. Fig. 6(a) shows a typical density distribution constructed by a pre-pulse of high-contrast laser irradiating on a 40-nanometer-thick foil target. Here, a laser pulse with a contrast ratio of about $10^{11}$, energy $\sim 1.2 \mathrm{~J}, 6 \mu \mathrm{m}$ FWHM focus diameter and peak intensity $2.14 \times 10^{20} \mathrm{~W} / \mathrm{cm}^{2}$ is used. For such density distribution, an exponential profile is more appropriate to describe the density distribution. Our additional simulations demonstrate that the vortex can also be generated in such density distribution as shown in Fig. 6.

The electron vortex distributions at two different instants are shown in Fig. 6(b,c). As one can see that similar vortices are generated and propagate transversely with a speed of about $0.36 c$. The energy dissipation and center position evolution of the vortex are given in Fig. 7. Although the vortices show similar dynamics, the energies carried by them in this case are much smaller. The optimal scale length of the density profile for the vortex generation and energy transportation still need to be studied in detail.

\section{Laser polarization effects}

The laser polarization effects on vortex generation and dynamics in 2D geometry are also investigated. Simulation results show that both linearly and circularly polarized laser pulses generate similar vortex structure once the laser intensities are the same, which is understandable since in the current scheme, the vortex generation is mainly related to the hot electrons' transportation around the laser exit region. The following vortex
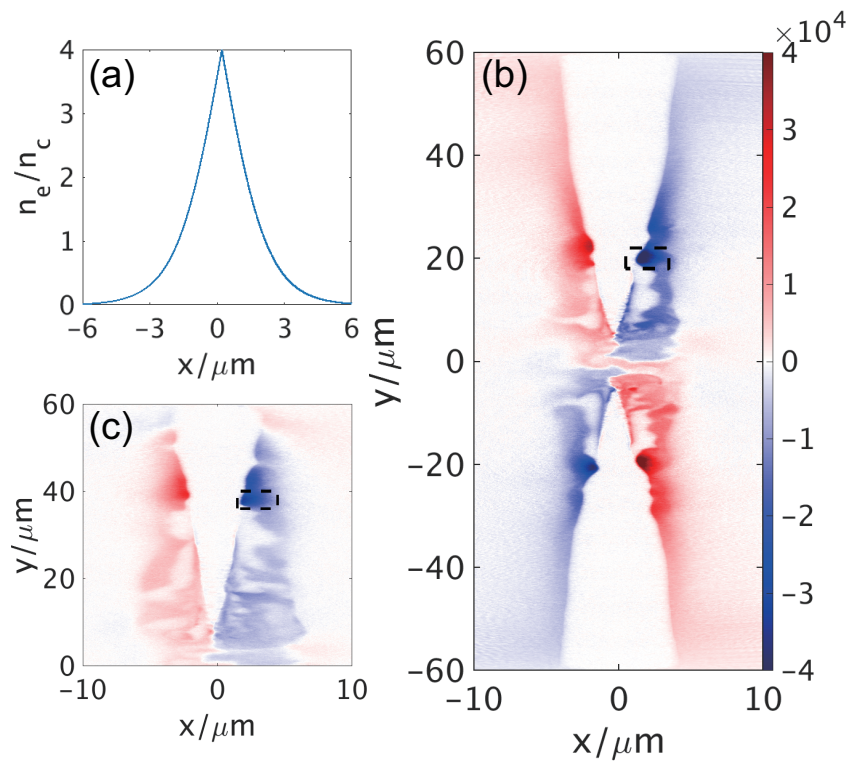

FIG. 6. (a) A Typical plasma density profile generated by a pre-pulse irradiating on a nanometer-thick ultra-thin foil. Distributions of magnetic dipole structures $\left(B_{z}\right.$ in the unit of Tesla) at the instants of $t=100 T_{0}$ (b) and $t=150 T_{0}$ (c).
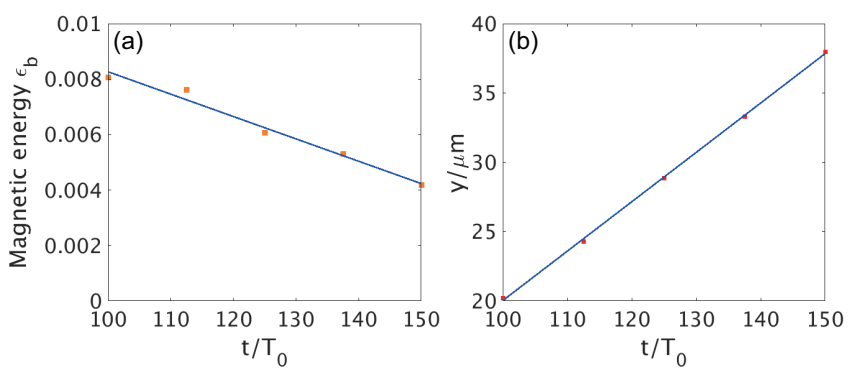

FIG. 7. (a) Evolution of the magnetic energy $\epsilon_{b}$ of the electron vortex in Fig. 6(a). (b) The center positions of the vortex at different instants.

transportation is self-sustained and does not depend on the laser pulse. The comparison of the generation and transportation of electron vortices in the cases by using lasers with different polarizations but same laser intensity is shown in Fig. 8(a-c). Both the vortex size, the magnetic field and the drift velocity are similar for the cases with p-, s- and circularly polarized drive pulses. For circularly polarized laser with double energy, the generated vortex is larger and faster. This is quantitatively understandable since both the charge and energy of the longitudinally emitted electrons at the downramp region of the plasma are higher. 


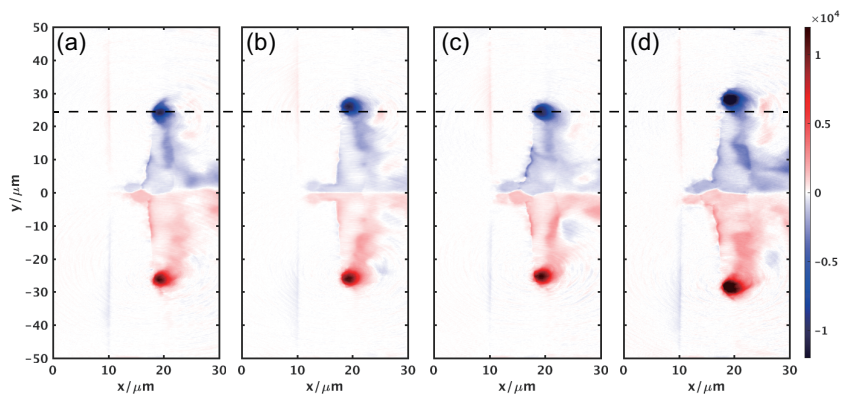

FIG. 8. (a) Distributions of magnetic fields of the electron vortices at $t=150 T_{0}$ driven by lasers with different polarizations (a-c). (a) p-polarization, $a_{0}=4.0$, (b) s-polarization, $a_{0}=4.0$, (c) circular polarization, $a_{0}=2.83$, and (d) circular polarization, $a_{0}=4.0$. Here the previous "plateaudownramp-plateau" density profile is used for all simulation cases.

\section{External magnetic field effects}

As we know in laser plasma interaction, intense static magnetic fields can be generated through many mechanisms $^{30-33}$. For this reason, we also studied the background or external magnetic field effects on the vortex dynamics. The results are shown in Fig. 9. The external magnetic field is along $+\vec{z}$ direction and is placed at the density down-ramp and the second plateau region as labeled in Fig. 9(a). One can see that with such intense external field $\vec{B}_{\text {ext }}$, one of the vortices whose magnetic field is along the same direction as $\vec{B}_{\text {ext }}$ moves faster than the other one. Simulations show that the moving velocity of each magnetic pole still obeys the analytical value, i.e., $\vec{V}_{\text {vortex }}=\left(\Omega_{z} \vec{z}+\vec{B}_{\text {ext }}\right) \times \nabla n_{e} / \mu_{0} n_{e}{ }^{2} e^{2}$ as long as $\vec{B}_{\text {ext }}$ is taken into account. The magnetic pole with opposite direction dissipates its magnetic energy to the ambient plasma and disappears later. As one can see from Fig. 9(a) and (b) that the upper vortex becomes smaller during its propagation while the bottom one almost keeps the same size. When an opposite external magnetic field is added, the Larmor radius of electrons in the vortex increases around the region where $\vec{B}+\vec{B}_{\text {ext }} \approx 0$ and these energetic electrons can run away from the vortex resulting in magnetic energy dissipation ${ }^{34}$. The magnetic energy of the upper vortex dissipates linearly with time as shown in Fig. 9(e). For the bottom vortex, the external magnetic field not only increases the speed of vortex motion, but also varies the vortex shape a little bit by elongating it along the $\mathrm{x}$ direction. This is because the balance between the forces induced by static electric and magnetic fields of the vortex is broken by adding the uniformly distributed external B field. These studies show that in a real laser plasma interaction case, to get a correct understanding of vortex dynamics, the background magnetic fields should be well considered. Meanwhile, it means that from vortex motion one can also deduce the information of background magnetic field.

\section{STRUCTURE AND DYNAMICS OF ELECTRON VORTEX IN THREE-DIMENSIONAL GEOMETRY}

The observed vortex structure can be generated when the target plasma has a slab profile, i.e. its transverse size is smaller than the laser focus spot. For a normal block plasma, such structure transforms to a $3 \mathrm{D}$ ring structure as shown in Fig. 10(a). A smoke-ring-like structure was observed inside the plasma in 3D simulation. The red isosurface shows the magnetic field along the ring. The projections on $x-y$ and $y-z$ planes show the plasma density distribution. The flow field of the $3 \mathrm{D}$ vortex is projected on $x-z$ plane. The streamlines around the vortex region are closed. We found that the ring expands radially along the direction of $\vec{B} \times \nabla n_{e}$ and the outward expansion velocity decreases along this expansion as shown in Fig. 10(b). The toroidal magnetic field $|\vec{B}|=B_{\theta}$ in the ring structure can be higher than 1000 Tesla when it is fully generated, but it drops when the ring expands. Since the typical expansion time scale is much larger than the electron's Larmor period, when discussing such ring expansion, the electron mass can be neglected in the fluid motion equation, i.e. $\vec{E}+\vec{V} \times \vec{B} \approx 0$. By using Faraday's law, the total volume integration of the toroidal magnetic field can be found to be constant, i.e. $\int_{\text {ring }} B_{\theta} d \tau=B_{\theta} 2 \pi R \pi r_{0}^{2}=$ const, where $R$ represents the radius of the ring and $r_{0}$ represents the radius of its cross section as labelled in Fig. 10(a). In the simulations we found $r_{0}$ does not vary so much. It means $B_{\theta} \times R=$ const, which is consistent with our simulation result until $t=85 T_{0}$ as shown in Fig. 10(c). Unlike in 2D vortex the magnetic field dissipation is mainly induced by ion motion, however, in 3D case both the structure expansion and the particle motion weaken the magnetic field. About $6 \%$ of the laser energy is transformed into the magnetic and eletric energies inside the vortex ring. They have been transported away along with the expansion of the vortex ring to a distance as far as 3 to 4 times large of the laser focus size. The magnetic field reduces quickly after $t=85 T_{0}$. In return, the weaker magnetic field makes the 3D ring expansion be slower at late time as shown in Fig. 10(b). As one can see from Fig.9 and the above observation that if there is an uniform external magnetic field $\vec{B}_{\text {ext }}$, the magnetic ring can be damaged since the side with the same direction of $\vec{B}_{\text {ext }}$ will be enhanced, while the opposite side will dissipate. In real experimental situation, self-generated magnetic fields by other mechanisms may lead to much more complicated structures of this magnetic ring.

\section{CONCLUSION AND DISCUSSION}

In conclusion, vortices and magnetic ring structures are found during ultrashort intense laser interaction with near critical density plasma. The current directly driven by the laser and the return current of background elec- 

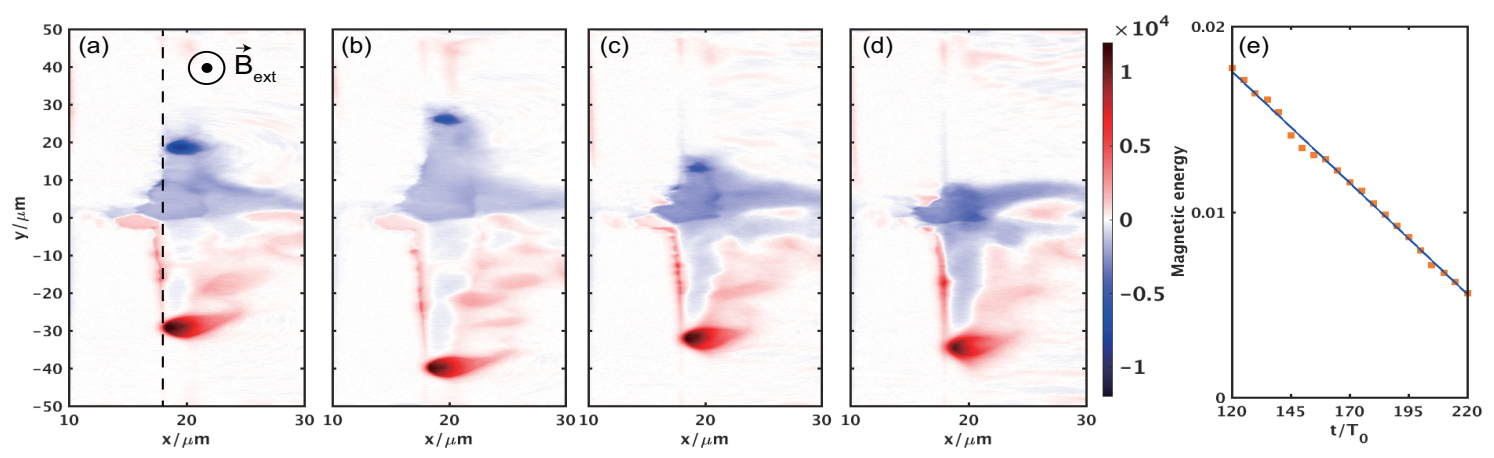

FIG. 9. Distribution of self-generated magnetic fields at the instant $t=t_{d}$ when an external magnetic field $\vec{B}_{\text {ext }}$ with different intensity is added in the simulation region. (a), (c) and (d) correspond to $t_{d}=150 T_{0}$, (b) corresponds to $t_{d}=200 T_{0}$. The external magnetic field is uniformly distributed in the region of the density down-ramp and the second plateau (i.e. the right side of the dashed line) and it is along $+\vec{z}$ direction as labeled in (a). (a, b) correspond to $\vec{B}_{\text {ext }}=1000 T$, (c) corresponds to $\vec{B}_{\text {ext }}=2000 T$, and (d) corresponds to $\vec{B}_{\text {ext }}=3000 T$. (e) The evolution of the magnetic field energy within the upper vortex region in the case of $\vec{B}_{\text {ext }}=1000 T$.
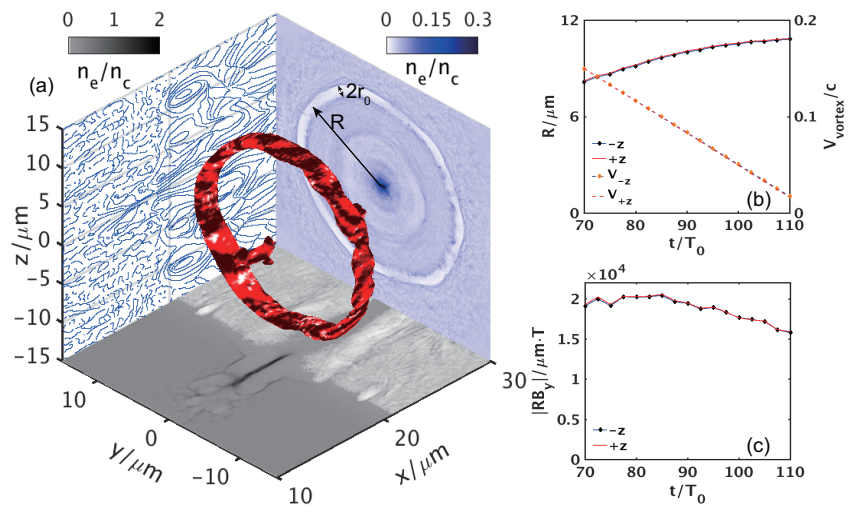

FIG. 10. (a) The smoke-ring-like structure of 3D electron vortex. The red isosurface shows the toroidal magnetic field with strength of $B_{\theta}=1.36 \times 10^{3} T$. Both $x-y$ and $y-z$ slices are cut through the center of the vortex. The flow field of the vortex is shown in $x-z$ plane. The evolution of the ring radius and the expansion velocity are shown in (b). The evolution of $B_{\theta} \times R$ are shown in (c). Here the magnetic fields $B_{\theta}(t)$ at the two vertices at $z= \pm R(t)$ are traced.

trons form a closed current, which firstly generates the local magnetic field. The density downramp introduces an asymmetric electric field. The combined fields lead to the transverse drift motion of the vortex. Using the EMHD model the dependence of vortex velocity on the plasma density gradient and the laser intensity are estimated and confirmed by PIC simulations. External or background magnetic fields with same direction as the vortex can enhance the vortex motion, the opposite magnetic fields slow down and weaken the vortex. In three dimensional geometry a smoke-ring-like magnetic structure is observed and the evolution of the toroidal magnetic field is investigated. These structures convert laser energy to magnetic energy and transport it far away. Beside laser-plasma interactions, vortices also exist in many magnetic activities in space science, such as KelvinHelmholtz instability induced vortices in Earth's magnetosphere where the plasma transportation and energy coupling are still primary unsolved questions (see Ref. ${ }^{35}$ and its references). Our findings provide a potential platform to study laboratory astrophysics by using ultrashort laser pulse with Joule level energy.

\section{ACKNOWLEDGEMENT}

The work was partly supported by NSFC (11991074, 11121504), NSAF (U1930111) of China, the Strategic Priority Research Program of Chinese Academy of Sciences (grant no. XDA25000000), the project High Field Initiative (CZ.02.1.01/0.0/0.0/15_003/0000449) from the European Regional Development Fund, and the U.S. DOE Office of Science Offices of HEP and FES (through LaserNetUS), under Contract No. DE-AC0205CH11231. Simulations were performed on the $\Pi$ supercomputer at Shanghai Jiao Tong University. The authors would like to acknowledge the OSIRIS Consortium, consisting of UCLA and IST (Lisbon, Portugal) for the use of OSIRIS and the visXD framework. The authors appreciate all the anonymous referees to provide very constructive suggestions to improve our studies.

\section{DATA AVAILABILITY}

The data that supports the findings of this study are available within the article and its supplementary material.

${ }^{1}$ C. Danson, C. Haefner, J. Bromage, T. Butcher, Jean-Christophe F. Chanteloup, E. A. Chowdhury, A. Galvanauskas, L. A. Gizzi, J. Hein, D. I. Hillier, et al., High Power Laser Sci. Eng. 7, E54 (2019). 
${ }^{2}$ D. Strickland and G. Mourou, Opt. Commun. 55, 447 (1985).

${ }^{3}$ S. V. Bulanov, M. Lontano, T. Zh. Esirkepov, F. Pegoraro, and A. M. Pukhov, Phys. Rev. Lett. 76, 3562 (1996).

${ }^{4}$ S. V. Bulanov, T. Zh. Esirkepov, M. Lontano, and F. Pegoraro, Plasma Phys. Rep. 23, 660 (1997).

${ }^{5}$ S. S. Bulanov, T. Zh. Esirkepov, F. F. Kamenets, and F. Pegoraro, Phys. Rev. E 73, 036408 (2006).

${ }^{6}$ L. M. Chen, H. Kotaki, K. Nakajima, J. Koga, S. V. Bulanov, T. Tajima, Y. Q. Gu, H. S. Peng, X. X. Wang, T. S. Wen, et al., Phys. Plasmas 14, 040703 (2007).

${ }^{7}$ K. V. Lezhnin, F. F. Kamenets, T. Zh. Esirkepov, S. V. Bulanov, Y. J. Gu, S. Weber, and G. Korn, Phys. Plasmas 23, 093116 (2016).

${ }^{8}$ K. V. Lezhnin, F. F. Kamenets, T. Zh. Esirkepov, and S. V. Bulanov, J. Plasma Phys. 84, 905840610 (2018).

${ }^{9}$ A. V. Kuznetsov, T. Zh. Esirkepov, F. F. Kamenets, and S. V. Bulanov, Plasma Phys. Rep. 27, 211 (2001).

${ }^{10}$ S. V. Bulanov, D. V. Dylov, T. Zh. Esirkepov, F. F. Kamenets, and D. V. Sokolov, Plasma Phys. Rep. 31, 369 (2005).

${ }^{11}$ S. V. Bulanov and T. Zh. Esirkepov, Phys. Rev. Lett. 98, 049503 (2007).

${ }^{12}$ Y. Fukuda, A. Ya. Faenov, M. Tampo, T. A. Pikuz, T. Nakamura, M. Kando, Y. Hayashi, A. Yogo, H. Sakaki, T. Kameshima, et al., Phys. Rev. Lett. 103, 165002 (2009).

${ }^{13}$ S. S. Bulanov, V. Y. Bychenkov, V. Chvykov, G. Kalinchenko, D. W. Litzenberg, T. Matsuoka, A. G. R. Thomas, L. Willingale, V. Yanovsky, K. Krushelnick, and A. Maksimchuk, Phys. Plasmas 17, 043105 (2010).

${ }^{14}$ T. Nakamura, S. V. Bulanov, T. Zh. Esirkepov, and M. Kando, Phys. Rev. Lett. 105, 135002 (2010).

${ }^{15}$ S. S. Bulanov, E. Esarey, C. B. Schroeder, W. P. Leemans, S. V. Bulanov, D. Margarone, G. Korn, and T. Haberer, Phys. Rev. ST Accel. Beams 18, 061302 (2015).

${ }^{16}$ D. Haberberger, S. Tochitsky, F. Fiuza, C. Gong, R. A. Fonseca, L. O. Silva, W. B. Mori, and C. Joshi, Nat. Phys. 8, 95 (2012).

${ }^{17}$ A. Sharma and A. Andreev, Laser Part. Beams 34, 219 (2016).

${ }^{18}$ H. Zhang, B. F. Shen, W. P. Wang, S. H. Zhai, S. S. Li, X. M. Lu, J. F. Li, R. J. Xu, X. L. Wang, X. Y. Liang, et al., Phys. Rev. Lett. 119, 164801 (2017).

${ }^{19}$ J. Park, S. S. Bulanov, J. Bin, Q. Ji, S. Steinke, J.-L. Vay, C. G. R. Geddes, C. B. Schroeder, W. P. Leemans, T. Schenkel, and E. Esarey, Phys. Plasmas, 26, 103108 (2019).
${ }^{20}$ Y. Y. Wang, F. Y. Li, M. Chen, S. M. Weng, Q. M. Lu, Q. L. Dong, Z. M. Sheng and J. Zhang, Sci. China-Phys. Mech. Astron. 60, 115211 (2017).

${ }^{21}$ L. Q. Yi, I. Pusztai, A. Pukhov, B. F. Shen, and T. Fülöp, J. Plasma Phys. 85, 905850403 (2019).

${ }^{22}$ D. J. Stark, T. Toncian, and A. V. Arefiev, Phys. Rev. Lett. 116, 185003 (2016).

${ }^{23}$ O. Jansen, T. Wang, D. J. Stark, E. d'Humières, T. Toncian, and A. V. Arefiev, Plasma Phys. Control. Fusion 60, 054006 (2018).

${ }^{24}$ J. R. Angus, A. S. Richardson, P. F. Ottinger, S. B. Swanekamp, and J. W. Schumer, Phys. Plasmas 21, 112306 (2014).

${ }^{25}$ A. S. Richardson, J. R. Angus, S. B. Swanekamp, P. F. Ottinger, and J. W. Schumer, Phys. Plasmas 20, 082115 (2013).

${ }^{26}$ L. I. Rudakov and J. D. Huba, Phys. Rev. Lett. 89, 095002 (2002).

${ }^{27}$ S. K. Yadav, A. Das, and P. Kaw, Phys. Plasmas 15, 062308 (2008).

${ }^{28}$ R. A. Fonseca, L. O. Silva, F. S. Tsung, V. K. Decyk, W. Lu, C. Ren, W. B. Mori, S. Deng, S. Lee, T. Katsouleas, and J.C.Adam, Lect. Notes Comput. Sci. 2331, 342 (2002).

${ }^{29}$ A. Hasegawa and K. Mima, Phys. Fluids 21, 87 (1978).

${ }^{30}$ J. A. Stamper, K. Papadopoulos, R. N. Sudan, S. O. Dean, E. A. McLean, and J. M. Dawson, Phys. Rev. Lett. 26, 1012 (1971).

${ }^{31}$ L. Gorbunov, P. Mora, and T. M. Antonsen, Jr., Phys. Rev. Lett. 76, 2495, (1996).

${ }^{32}$ Z. M. Sheng and J. Meyer-ter-Vehn, and A. Pukhov, Phys. Plasmas 5, 3764 (1998).

${ }^{33}$ S. Y. Zhou, Y. F. Bai, Y. Tian, H. Y. Sun, L. H. Cao, and J. S. Liu, Phys. Rev. Lett. 121, 255002, (2018).

${ }^{34}$ M. Hata, H. Sakagami, and A. Das Phys. Plasmas 20, 042303 (2013).

${ }^{35}$ H. Hasegawa, M. Fujimoto, T. -D. Phan, H. Rème, A. Balogh, M. W. Dunlop, C. Hashimoto, and R. TanDokoro, Nature 430, 755 (2004).

${ }^{36}$ A. Yogo, H. Daido, S. V. Bulanov, K. Nemoto, Y. Oishi, T. Nayuki, T. Fujii, K. Ogura, S. Orimo, A. Sagisaka, et al., Phys. Rev. E. 77, 016401 (2008).

${ }^{37}$ A. A. Andreev, S. Steinke, T. Sokollik, M. Schnürer, S. T. Avetsiyan, K. Y. Platonov, and P. V. Nickles, Phys. Plasmas 16 , 013103 (2009). 